\title{
The sophrology as adjuvant therapy may improve the functionality of patients with fibromyalgia. A pilot randomized controlled trial
}

Franciele Santos de Oliveira', Hugo Jario de Almeida Silva', Jaine Maria de Pontes Oliveira', José Felipe Costa da Silva', Valéria Lidyanne Silva Gomes¹, Diogo Simões Fonseca², Caio Alano de Almeida Lins³ ${ }^{3}$ Marcelo Cardoso de Souza ${ }^{4}$

\begin{abstract}
Introduction: Sophrology is the science of the serene spirit applied to human consciousness. Body relaxation exercises are not yet a consensus in the treatment of fibromyalgia. Objective: To investigate the effectiveness of a relaxation exercise program based on sophrology in patients with fibromyalgia. Method: This is a pilot study, controlled, randomized and with blinded evaluator and eighteen patients with medical diagnosis of fibromyalgia were randomized into two groups: the intervention group (IG) who participated in sophrology sessions $(n=8)$ and control group (CG) who participated of a resistance training program $(n=10)$. Both groups underwent treatments 2 times per week for 12 weeks and reevaluations every 4 weeks. The assessment instruments used were the visual analogic scale of pain (VAS), the satisfaction with the treatment by the Likert scale, the 6-minute walk test (6MWT), the Timed Up and Go test (TUG) and the Fibromyalgia Impact Questionnaire (FIQ). Results: We did not find statistical differences in any of the variables between the groups. The intragroup evaluation, there was a significant difference in CG for TC6 $(p=0.01)$ and TUG $(p=0.04)$. In IG there was a statistically significant difference $(p=0.02)$ for the FIQ. Conclusion: Thus, the improvement in 6MWT and TUG in the CG and the improvement in the FIQ in the IG group showed promising results, although studies with larger sample is needed for more accurate results.
\end{abstract}

Keywords: Sophrology. Physiotherapy. Fibromyalgia.

\section{INTRODUCTION}

The importance of physical exercise for patients with fibromyalgia has already been well founded in the literature. In addition to being a low-cost, safe and efficient intervention for the treatment of fibromyalgia syndrome, exercise is also effective in reducing pain and number of pain points, improving quality of life, mood and other psychological aspects. ${ }^{(1,2)}$ Although there are benefits in almost all exercise modalities, further evidence supports the practice of aerobic training..$^{(1,3)}$

Studies have shown that patients with fibromyalgia have reduced muscle strength in the lower and upper limbs, in this sense, recent studies show that muscle strengthening or resistance training should be recommended in this condition. ${ }^{(4-7)}$

Body relaxation exercises also seem to show some beneficial effect but there is no consensus on this, since we always recommend more active than passive exercise. Techniques such as yoga, tai chi, acupuncture and music are being cited in the literature as complementary alternatives with promising effects. ${ }^{(8-10)}$
Among the exercises of corporal relaxation, sophrology that is well known in European countries, has been gaining space in the Brazilian national scene. sophrology originates from the words: sos= serenity, harmony; Phren= mind, consciousness; and logos= study, knowledge. Thus, etymologically "sophrology" means: study of consciousness in equilibrium. It is the science of the serene spirit applied to the human consciousness. ${ }^{(11)}$

\begin{abstract}
"Sophrology is not hypnosis, it is not psychoanalysis, it is no longer a relaxation technique, it does not require a religion: Sophrology is secular and apolitical. It has nothing to do with suffering, it is a true invitation to well-being. The method consists of a series of dynamic and static relaxation techniques, breathing exercises, physical movements, and positive thinking strategies that broaden the field of consciousness, particularly with regard to body perception and strengthening of the body-spirit relationship. It aims to work on self-confidence, concentration, adaptability, autonomy, intrinsic value of the individual, physical and mental harmony, the ability to build projects and achieve
\end{abstract}

Corresponding Author: Marcelo Cardoso de Souza. Rua Vila Trairi, S/N, Centro, Santa Cruz - RN, CEP: 59200-000. Telephone: +55 (84) 3291.2411.

E-mail: marcelocardoso@facisa.ufrn.br

4- Physiotherapy course, Academic Master's in Collective health, "Faculdade de Ciências da Saúde do Trairi", "Universidade Federal do Rio Grande do Norte" (FACISA/UFRN). Santa Cruz (RN), Brazil.

Full list of author information is available at the end of the article.

Financial support: The authors declare they were financed by Edital 03/2015 - PROPESQ_NUPLAM_2015-2016/UFRN.

Submission date 23 August 2016; Acceptance date 1 December 2016; Publication online date 15 December 2016 
a satisfactory performance in terms of personal and professional performance, positive attitude towards stress, creative vision of how to relate to the world and optimism about the future and the tranquility within the present". ${ }^{(12)}$

The use of sophrology at a therapeutic level is reserved for health professionals who can use the technique as a single or complementary therapy to increase the patient's resistance, achieving a more serene and tolerant attitude towards aggressions caused by the disease. ${ }^{(13)}$

This study arose from the need to study a new intervention for a well-known disease and that practically fill the public health services, rehabilitation centers, looking for physiotherapeutic treatment as in our service in Santa Cruz, Rio Grande do Norte.

The objective of the study was to evaluate the effectiveness of a sophrology-based relaxation exercise program, compared to a control group that performed resistance exercises.

\section{METHOD}

This is a pilot, controlled, randomized, blind-valued trial. Eighteen patients with medical diagnosis of fibromyalgia were randomized into two groups. The intervention group (IG) who participated in sophrology sessions $(n=8)$ and control group (CG) who participated of a resistance training program $(n=10)$. Both groups underwent treatments 2 times per week for 12 weeks. The randomization was done through a computer program, and from this result, sheets were made and placed in opaque and sealed envelopes to maintain allocation secrecy.

The study was approved by the CEP/FACISA (Number: 1.245.074) and was carried out at Clinic School of Physiotherapy from "Faculdade de Ciências da Saúde do Trairi" in the "Universidade Federal do Rio Grande do Norte" - FACISA/UFRN, Santa Cruz-RN.

Patients of the female gender with fibromyalgia diagnosis were included, with medical referral and according to the classification criteria of the American College of Rheumatology, aged 18 to 60 years. All patients read and signed the informed consent form.

Excluded patients were the ones with: uncontrolled arterial hypertension, decompensated cardiorespiratory disease, history of syncope or arrhythmias induced by physical exercise, decompensated diabetes, serious psychiatric illness, history of regular physical exercise (at least 2 times a week) in the last 6 months and any other condition that would make it impossible for the patient to perform physical exercises.

\section{Intervention group}

IG performed 2 sessions of body relaxation based on the technique of sophrology per week, lasting 40 minutes for 12 weeks. Patients were lying on mats, comfortable, with relaxing music in the background in a room with pleasant temperature. A physiotherapist recited some sentences, as shown in figure 1.

Upon entering this relaxation, the patients were invited to think a little about their illness, their life, imagining the positives and negatives and when analyzing everything, the physiotherapist requested that they focus on the negative points and when focusing on these negative points, it was request that they try to see a good side of each point. At that moment, the patients returned from relaxation through a command "Tighten your hands tightly, the calmer and relaxed, the stronger we will shake hands, now we will have a beautiful stretch as we do in the morning".

\section{Control Group}

GC performed a resistance training program on a weight training apparatus by calculating 1 maximal repetition (1MR), twice a week, lasting 40 minutes for a period of 12 weeks. The program of exercises is described below:

$\checkmark 3$ sets of 12 repetitions, with an interval of 1 to 2 minutes of recovery from one series to another, alternating upper and lower limbs.

$\checkmark 60 \%$ of $1 \mathrm{RM}$ in the first month, $70 \%$ of $1 \mathrm{RM}$ in the second month and $80 \%$ of 1 RM in the third month.

$\checkmark$ At the end of every 4 weeks, the patients were reevaluated for progression of the loads.

$\checkmark$ The following muscles were trained through resistance exercises: quadriceps femoris, hamstrings, hip abductors and adductors, biceps brachii, triceps, pectorals and trapezius.

"close your eyes", "analyze your breathing and your heartbeat in order to
reassure them", "imagine your body progressively relaxing, muscle to muscle",
"I'm feeling my left arm well relaxed, super relaxed, more and more relaxed",
"imagine a river in a forest", "feel the waters of this river passing between your
feet", "these waters are withdrawing all the pain, fatigue..."

Figure 1. Main sentences given to patients in the intervention group. 
The evaluations were performed by a blind physiotherapist at the following times: initial evaluation (T0), 4 weeks (T4), 8 weeks (T8) and at 12 weeks (T12). The outcomes evaluated were:

- Pain (T0, T4, T8 and T12): Through the visual analogue scale (VAS), where the patient indicated the degree of his pain, with a score of 0 to $10 \mathrm{~cm}$, zero being painless and ten unbearable pain. ${ }^{(14)}$

- Patient satisfaction with the treatment (T4, T8 and T12): The patients were questioned in the second evaluation with respect to treatment satisfaction by a Likert scale of 1 to 5, in which: 1 - much worse; 2 - a little worse; 3- unchanged; 4- a little better; 5- much better. ${ }^{(15)}$

\section{- Functional Performance (T0 and T12):}

o The six-minute walk test (6MWT) was performed on a 22-meter indoor track, following the guidelines of American Thoracic Society (ATS). ${ }^{(16)}$

o The Timed Up and Go (TUG) is a functional test that quantifies the functional mobility in seconds through the time the individual performs the task, i.e., how many seconds does he lift from a standard chair with support and arms (approximately $46 \mathrm{~cm}$ of height and arms of $65 \mathrm{~cm}$ in height), walks 3 meters, turns, returns towards the chair and sits again. The test was designed primarily to evaluate predictors of falls in the elderly, with the following scores: less than 20 seconds for performance, corresponding to low risk for falls, 20 to 29 seconds, medium risk for falls and 30 seconds or more, a high risk for falls. ${ }^{(17)}$

- Quality of life related to disease (TO and T12): The evaluation was performed through the Fibromyalgia Impact Questionnaire (FIQ), questionnaire that evaluates anxiety, depression and physical fitness in fibromyalgia. The translated version was used for the Brazilian population. ${ }^{(18)}$

The program used for the analysis was Statistical Package for Social Sciences - SPSS, version 20.0. The Student's t-test for independent samples was applied for analysis between groups and the paired t-test for comparison of variables $6 \mathrm{MWT}$, TUG and FIQ before and after the intervention. The ANOVA was performed to compare the EVA and Likert variables with post hoc Bonferroni. The mean differences were considered statistically significant when $p<0.05$.

\section{RESULTS}

The groups presented homogenous initial characteristics, with no significant difference ( $p>0.05$ ) to the age, $C G=42.4 \pm 14.0$ and $I G=46.3 \pm 10.6$ years and to the body mass index (BMI) $29.0 \pm 5.5$ and $27.6 \pm 5.4 \mathrm{~kg} / \mathrm{m}^{2}$, respectively.

The table below shows the mean values obtained for VAS and Likert scale in the 12-week intervention period. Although a significant difference, $p=0.02$, for the VAS in the control group, adjustment by the Bonferroni test did not show a statistical difference between the moments of intervention, $\mathrm{p}=0.14$ (table 1 ).

The Likert scale was applied at three moments of the intervention period, and did not show significant variation, $p=0.23$, during the 12 -week period.

The results of the 6MWT presented expressive gains in both groups; however, there was no significant difference between the initial and final moments after 12 weeks between the groups. Both TUG and FIQ presented the same results obtained in the 6MWT, indicating that there was no statistically significant difference between the initial and final moments between the groups (table 2). However, when comparing the initial and final moments, intra-group by t test for paired samples, a significant difference was observed in the CG to the 6MWT ( $p=0.01)$ and TUG $(p=0.04)$ variables. In the IG was observed a statistically significant difference $(p=0.02)$ to the FIQ.

\section{DISCUSSION}

It is important to observe the behavior of the two groups in relation to the likert scale, since the two groups behaved equally, with no difference between them, showing that patients in both groups reported the same degree of satisfaction with the proposed treatment.

The improvement of the control group that performed the resisted exercises in relation to pain improvement and

Table 1. Results for the evolution of pain scales (VAS) and Likert scale satisfaction after the 12-week intervention of both groups.

\begin{tabular}{|c|c|c|c|c|}
\hline \multirow{3}{*}{ Weeks } & \multicolumn{2}{|c|}{ CG (resistance exercise) } & \multicolumn{2}{|c|}{ IG (sophrology) } \\
\hline & $\operatorname{VAS}(n=10)$ & Likert $(n=10)$ & $\operatorname{VAS}(n=8)$ & Likert $(n=8)$ \\
\hline & Mean $\pm S D$ & Mean \pm SD & Mean $\pm S D$ & Mean $\pm S D$ \\
\hline $\mathrm{T}_{0}$ & $6.50 \pm 1.72$ & --- & $5.25 \pm 1.39$ & --- \\
\hline $\mathrm{T}_{4}$ & $5.70 \pm 3.30$ & $4.44 \pm 0.53$ & $4.75 \pm 2.05$ & $4.29 \pm 0.49$ \\
\hline $\mathrm{T}_{8}$ & $5.60 \pm 2.41$ & $4.56 \pm 0.53$ & $3.87 \pm 2.10$ & $4.57 \pm 0.54$ \\
\hline$T_{12}$ & $3.70 \pm 2.87^{*}$ & $4.33 \pm 0.50$ & $4.13 \pm 1.96$ & $4.43 \pm 0.54$ \\
\hline
\end{tabular}

\footnotetext{
$* p<0.05$ in the intragroup evaluation. Statistical test used: Anova of repeated measurements. VAS= Visual Analog Scale of Pain.
} 
Table 2. Description of results after 12 weeks of resistance training (CG) and of sophrology (IG) in patients with fibromyalgia.

\begin{tabular}{|c|c|c|c|c|c|c|}
\hline \multirow{2}{*}{ Weeks } & \multicolumn{2}{|c|}{ 6MWT } & \multicolumn{2}{|c|}{ TUG } & \multicolumn{2}{|c|}{ FIQ } \\
\hline & то & T12 & то & T12 & то & T12 \\
\hline CG & $393.6 \pm 52.0$ & $453.9 \pm 62.8^{*}$ & $13.2 \pm 3.2$ & $9.4 \pm 3.2 *$ & $9.4 \pm 2.7$ & $8.5 \pm 2.1$ \\
\hline IG & $424.4 \pm 58.8$ & $449.9 \pm 71.6$ & $12.8 \pm 2.0$ & $10.8 \pm 2.7$ & $10.4 \pm 2.0$ & $8.3 \pm 2.8^{*}$ \\
\hline
\end{tabular}

${ }^{*} \mathrm{p}<0.05$ in the intragroup evaluation. CG= control group $(\mathrm{n}=8), \mathrm{IG}=$ intervention group $(\mathrm{n}=10), 6 \mathrm{MWT}=6$-minute walk test, TUG= Timed Up and Go teste, FIQ= Fibromyalgia Impact Questionnaire.

the 6MWT and TUG, allows us to infer that trained muscles provided greater distance of walking and better time in the TUG, although this improvement was observed only within the same group.

We believe that functional improvement assessed by the FIQ of sophrology group, even intra-group, is motivating and suggestive for further studies.

Sophrology has also been used in other rheumatic diseases such as rheumatoid arthritis ${ }^{(19)}$ and in the elderly to reduce pain and anxiety. ${ }^{(20)}$

Non-pharmacological treatment is an important part of treatment, and there is evidence supporting a range of interventions, including aerobic exercise, strength training, stretching, cognitive-behavioral therapy, and patient education. Alternative and complementary medicine techniques have not yet been fully recognized by scientific medicine because little is known about their mechanisms of action and utility. ${ }^{(21)}$

According to Braz et al. ${ }^{(22)}$ it is important that physicians acquire knowledge about other forms of complementary therapies, whether or not they are used, and that they can dialogue with their patients and guide them about these forms of treatment, since recent reviews have shown positive results for some techniques which are part of complementary and alternative medicine. ${ }^{(23)}$

The study presents some limitations such as small sample size, so it was considered a pilot study, although the design adopted as a clinical, controlled and randomized trial with blind evaluator is the most appropriate for studies comparing interventions.

\section{CONCLUSION}

We found improvement of pain, functional improvement by $6 \mathrm{MWT}$ and TUG, intra-group, in patients with fibromyalgia of the control group who performed resistance exercises. The patients in the intervention group who underwent sophrology, obtained functional improvement evaluated through FIQ. There was no difference in any of the variables in the analysis between groups.

Thus, we should encourage studies with a greater number of patients and a deeper understanding of this technique to allow a wider range of therapeutic options in the treatment of fibromyalgia, knowing that sophrology can present promising results as a complementary treatment for patients with fibromyalgia.

\section{AUTHOR'S CONTRIBUTIONS}

Franciele Santos de Oliveira, Hugo Jario de Almeida Silva, Jaine Maria de Pontes Oliveira, José Felipe Costa da Silva, Valéria Lidyanne Silva Gomes, Diogo Simões Fonseca, Caio Alano de Almeida Lins: Data collection, manuscript preparation, final review; Marcelo Cardoso de Souza: Interpretation of data, study orientation, final review.

\section{CONFLICT OF INTEREST}

The authors declare that they have no conflicts of interest.

\section{AUTHOR DETAILS}

1- physiotherapy course, "Faculdade de Ciências da Saúde do Trairi", "Universidade Federal do Rio Grande do Norte" (FACISA/UFRN). Santa Cruz (RN), Brazil.

2- Physiotherapy Department, "Universidade Federal de Juiz de Fora- Campus Avançado de Governador Valadares" (UFJF-GV), (MG), Brazil.

3- physiotherapy course and post-graduation program in rehabilitation sciences, "Faculdade de Ciências da Saúde do Trairi", "Universidade Federal do Rio Grande do Norte" (FACISA/UFRN). Santa Cruz (RN), Brazil.

\section{REFERENCES}

1- Valim V, Natour J, Xiao Y, Pereira AF, Lopes BB, Pollak DF, et al. Effects of physical exercise on serum levels of serotonin and its metabolite in fibromyalgia: a randomized pilot study. Rev Bras Reumatol. 2013; 53 (6): 538-41.

2- Bidonde J, Busch AJ, Bath B, Milosavljevic S. Exercise for adults with fibromyalgia: an umbrella systematic review with synthesis of best evidence. Curr Rheumatol Rev. 2014; 10: 45-79.

3- Valim V, Oliveira L, Suda A, Silva L, de Assis M, Barros Neto T, et al . Aerobic fitness effects in fibromyalgia. J Rheumatol. 2003; 30 (5): 1060-9.

4- Jorge RT, Souza MC, Jones A, Júnior IL, Jennings F, Natour J. Treinamento resistido progressivo nas doenças musculoesqueléticas crônicas. Rev. Bras. Reumatol. 2009; 49 (6): 726-734.

5- Cardoso FS, Curtolo M, Natour J, Lombardi Jr I. Avaliação da qualidade de vida, força muscular e capacidade funcional em mulheres com fibromialgia. Rev. Bras. Reumatol. 2011; 51 (4): 344-350.

6- Larsson A, Palstam A, Löfgren M, Ernberg M, Bjersing J, Bileviciute-Ljungar I, et al. Resistance exercise improves muscle strength, health status and pain intensity in fibromyalgia-a randomized controlled trial. Arthritis Res Ther. 2015; 17:161.

7- Jones KD, Burckhardt CS, Clark SR, Bennett RM, Potempa KM. A randomized controlled trial of muscle strengthening versus flexibility training in fibromyalgia. J Rheumatol. 2002; 29: 1041-8.

8- da Silva GD, Lorenzi-Filho G, Lage LV. Effects of yoga and the addition of Tui $\mathrm{Na}$ in patients with fibromyalgia. J Altern Complement Med. 2007;13 (10): 1107-13.

9- Garza-Villarreal EA, Wilson AD, Vase L, Brattico E, Barrios FA, Jensen $\mathrm{TS}$, et al. Music reduces pain and increases functional mobility in fibromyalgia. Front Psychol. 2014; 11: 5-90.

10- Wang C, McAlindon T, Fielding RA, Harvey WF, Driban JB, Price LL, et al. A novel comparative effectiveness study of Tai Chi versus aerobic exercise for fibromyalgia: study protocol for a randomized controlled trial. Trials. 2015; $16: 34$. 
11- Bonnet W. Introdução à sofrologia. Neurociências. 2010; 6 (1): 63-73.

12- Cruz AV, Viegas MH. Escola de Sofrologia Caycediana de Santarém. Disponível em http://www.sofrologia.pt/Escola_de_Sofrologia_ Caycediana_de_Santarem/O_que_e.html. Acesso 10/01/2016

13- Bonnet W. Portal A Sofrologia. Disponível em http://www.sophrologie. net/a-sofrologia/?lang=pt-br. Acesso em 10/01/2016.

14- Maeda C; Martinez JE; Neder M. Efeito da Eutonia no Tratamento da Fibromialgia. Rev Bras Reumatol. 2006; 46 (1): 3-10.

15- Drinkwater BL. A comparison of the direction-of-perception technique with the Likert method in the measurement of attitudes. J Soc Psychol. 1965; 67 (2): 189-96.

16- Brooks D, Solway $S$, Gibbons WJ. ATS statement on six-minute walk test. Am J Respir Crit Care Med. 2003; 167(9): 1287.

17- Podsiadlo D, Richardson S. The timed "Up \& Go": a test of basic functional mobility for frail elderly persons. J Am Geriatr Soc. 1991; 39 (2): 142-8.
18- Marques AP; Santos AMB; Assumpção A; Matsutani LA; Lage LV; Pereira CAB. Validação da versão brasileira do fibromialgia impacto questionário (FIQ). Rev. Bras. Reumatol. 2006; 46 (1): 24-31.

19- Guillemot G. Sophrology and rheumatoid arthritis. Soins. 2004;(688):46.

20- Tocheport P. Sophrology in geriatrics, an innovative approach to reducing pain and anxiety. Soins. 2012 Mar;(763):16-20.

21- Cassisi G, Ceccherelli F, Atzeni F, Sarzi-Puttini P. Complementary and alternative medicine in fibromyalgia: a practical clinical debate of agreements and contrasts. Clin Exp Rheumatol. 2013; 31(6 Suppl 79): S134-52.

22- Braz AS, Paula AP, Diniz M, Almeida R. Uso da terapia não farmacológica, medicina alternativa e complementar na fibromialgia. Rev. Bras. Reumatol. 2011; 51 (3): 275-282.

23- Lauche R, Cramer H, Häuser W, Dobos G, Langhorst J. A Systematic Overview of Reviews for Complementary and Alternative Therapies in the Treatment of the Fibromyalgia Syndrome. Evid Based Complement Alternat Med. 2015;2015:610615. doi: 10.1155/2015/610615. Epub 2015 Jul 13. 